\title{
Evaluation of the proliferation and differentiation behaviors of mesenchymal stem cells with partially converted borate glass containing different amounts of strontium in vitro
}

\author{
YI ZHU $^{1 *}$, YUANMING OUYANG $^{1 *}$, YI CHANG $^{2}$, CONGFENG LUO $^{1}, \mathrm{JUN} \mathrm{XU}^{1}$, \\ CHANGQING ZHANG $^{1}$ and WENHAI $\mathrm{HUANG}^{3}$
}

Departments of ${ }^{1}$ Orthopaedic Surgery and ${ }^{2}$ Plastic Surgery, Shanghai Sixth People's Hospital, Shanghai Jiaotong University, Shanghai 200233; ${ }^{3}$ Institute of Bioengineering and Information Technology Materials, Tongji University,

Shanghai 200092, P.R. China

Received November 27, 2012; Accepted January 22, 2013

DOI: $10.3892 / \mathrm{mmr} .2013 .1341$

\begin{abstract}
The objective of this study was to examine the proliferation and differentiation behaviors of different compositions of strontium-containing (from 0-12 mol\%) borate glasses with mesenchymal stem cells (MSCs). The Cell Counting Kit-8 (CCK-8) assay revealed that after three days of culturing, the 6Sr group had the highest cell growth rate. Analysis of cell morphology revealed that cells proliferated well near the particles of the samples in all the groups on day 3 . On day 7 , cells in the $6 \mathrm{Sr}$ group demonstrated a higher proliferation rate than other 4 groups under the microscope. When performing the Live-Dead staining experiment, the $6 \mathrm{Sr}$ group had the least number of dead cells. Total DNA qualification indicated that the $6 \mathrm{Sr}$ group had a statistically higher concentration compared with the remaining groups. It was found that on day 7, compared with the 0Sr group, the core binding factor $\alpha 1$ (Cbfa1) mRNA expression level was significantly higher in the $6 \mathrm{Sr}, 9 \mathrm{Sr}$ and $12 \mathrm{Sr}$ groups. On day 14 , compared with the $0 \mathrm{Sr}$ group, the bone sialoprotein (BSP) mRNA level was significantly higher in the 6Sr group. Additionally, on day 21, the 6Sr and 9Sr groups demonstrated higher osteocalcin (OCN) mRNA expression levels compared with the 0Sr group. In the alkaline phosphatase (ALP) activity test, on day 21 , the $6 \mathrm{Sr}$ group presented a higher activity than the $0 \mathrm{Sr}$ group. Further, the number of mineralized nodules per unit in MSCs was measured by Alizarin Red
\end{abstract}

Correspondence to: Dr Jun Xu, Department of Orthopaedic Surgery, Shanghai Sixth People's Hospital, Shanghai Jiaotong University, 600 Yishan Road, Shanghai 200233, P.R. China

E-mail: junxu19781214@gmail.com

${ }^{*}$ Contributed equally

Key words: borate glass, strontium, proliferation, differentiation, mesenchymal stem cell
$\mathrm{S}$ staining. The results showed that the $6 \mathrm{Sr}$ and $9 \mathrm{Sr}$ groups had the greatest number of mineralized nodules. Therefore, it could be concluded that borate glasses containing strontium oxide of $0,3,6,9$ and $12 \mathrm{~mol} \%$ demonstrate a significant level of proliferation when interacting with MSCs. The borate glass containing 6 mol\% strontium oxide had the greatest level of proliferation when cultured with MSCs. The borate glass containing 6 and 9 mol\% strontium oxide facilitated an improved bone formation ability compared with the remaining two compositions.

\section{Introduction}

Bioactive glasses have attractive behaviors for biomedical substitutes, and provide a biological alternative for bone and tissue regeneration $(1,2)$. For example, silicate-based bioglass, such as 45S5 glass (3), has been most widely studied for its biomedical applications (4). Due to its incomplete degradation behaviors (5), 45S5 glass has gradually been replaced by the bioactive borate glass (6), which has a similar composition to $45 \mathrm{~S} 5$ glass, but all the $\mathrm{SiO}_{2}$ is replaced with $\mathrm{B}_{2} \mathrm{O}_{3}$. The new composition has a faster degradation ability and a higher conversion rate than silicate 45S5 glass. It has been suggested that borate glass could be applied in the field of tissue engineering $(7,8)$.

To the best of our knowledge, strontium is a trace element in humans and has been applied as a medication, such as strontium ranelate in the treatment of osteoporosis. $\mathrm{Sr}^{2+}$ has been found to promote osteogenic differentiation of mesenchymal stem cells (MSCs) (9-11), stimulate osteoblast proliferation $(12,13)$ and accelerate osteoclast apoptosis (14). Moreover, incorporating strontium into bioactive glass improves the bioactivity of bone repair (15) and bone formation (16). Therefore, we hypothesized that strontium-containing bioactive borate glass would have improved cell compatibility in tissue engineering.

The purpose of our study was to evaluate the proliferation and differentiation effects of strontium in borate glass on MSCs in vitro. Additionally, we attempted to determine an appropriate composition of strontium in borate glass for future applications in vivo. 
Table I. Composition of the groups (mol\%).

\begin{tabular}{lcccccccc}
\hline Group & $\mathrm{Na}_{2} \mathrm{O}$ & $\mathrm{K}_{2} \mathrm{O}$ & $\mathrm{MgO}$ & $\mathrm{CaO}$ & $\mathrm{SrO}$ & $\mathrm{SiO}_{2}$ & $\mathrm{~B}_{2} \mathrm{O}_{3}$ & $\mathrm{P}_{2} \mathrm{O}_{5}$ \\
\hline A: $0 \mathrm{Sr}$ & 6 & 8 & 8 & 22 & 0 & 18 & 36 & 2 \\
B: $3 \mathrm{Sr}$ & 6 & 8 & 5 & 22 & 3 & 18 & 36 & 2 \\
C: $6 \mathrm{Sr}$ & 6 & 8 & 2 & 22 & 6 & 18 & 36 & 2 \\
D: $9 \mathrm{Sr}$ & 6 & 8 & 0 & 21 & 9 & 18 & 36 & 2 \\
E: $12 \mathrm{Sr}$ & 6 & 8 & 0 & 18 & 12 & 18 & 36 & 2 \\
\hline
\end{tabular}

\section{Materials and methods}

\section{Sample preparation}

Biomaterials synthesis. The preparation of the glass samples by melting and casting processes was performed as described previously $(16,17)$. Generally, the glass samples with the composition of $\mathrm{Na}_{2} \mathrm{O}-\mathrm{K}_{2} \mathrm{O}-\mathrm{MgO}-\mathrm{CaO}-\mathrm{B}_{2} \mathrm{O}_{3}-\mathrm{SiO}_{2}-\mathrm{SrO}-\mathrm{P}_{2} \mathrm{O}_{5}$ (Table I) were prepared by melting each analytical chemical in a platinum crucible at $1,500^{\circ} \mathrm{C}$ for $2 \mathrm{~h}$ with stirring, and then quenching the melt between cold stainless steel plates into glass flakes. These flakes were used as glass wafers (1-mm thick) in the assay experiments, while some of the glass flakes were crushed into fine particles (105-125 $\mu \mathrm{m}$ in size) and used for the extraction experiments.

Prior to all the experiments, the particles or flakes were soaked in $0.025 \mathrm{M} \mathrm{K}_{2} \mathrm{HPO}_{4}$ solution for 10 days to allow for the superficial reaction and to avoid the fast borate-releasing stage occurring during the following the experimental processes $(18,19)$. Scanning electron microscope (SEM) observation was performed following the soaking of samples and the partial conversion to hydroxyapatite on the surface of borate glass particles, and prior to commencing the experiments (Fig. 1).

Sample degradation. Assessment of the in vitro degradation of strontium in borate glass for each group was conducted by statically immersing the glass particles of each group $(1 \mathrm{~g})$ in $0.02 \mathrm{M} \mathrm{K}_{2} \mathrm{HPO}_{4}$ solution $(100 \mathrm{ml} ; \mathrm{pH} 7.0)$ at $37^{\circ} \mathrm{C}$ for 28 days. The strontium in the immersion solution at different time points was analyzed by ion chromatography (DX 500 Dionex, USA). The average values were obtained from three parallel experiment samples. The accumulated released irons of strontium at different time points were calculated by the weight of the strontium in the solution divided by that in the original samples.

MSC culture. Canine bone marrow-derived mesenchymal stem cells (MSCs) were used in the present experiment. The animal study was approved by the Committee of Medical Ethics and the Institutional Review Board of Shanghai Sixth People's Hospital, Shanghai Jiaotong University (China). MSCs were isolated by density gradient centrifugation following iliac bone marrow aspiration. The cells were resuspended in Dulbecco's modified Eagle's medium (DMEM; Gibco-BRL, Carlsbad, CA, USA) supplemented with $10 \%$ fetal bovine serum (FBS; Thermo Scientific, HyClone, Logan, UT, USA), $100 \mathrm{IU} / \mathrm{ml}$ penicillin (Gibco-BRL) and $100 \mathrm{lg} / \mathrm{ml}$ streptomycin (Gibco-BRL), and cultured at $37^{\circ} \mathrm{C}$ in $5 \% \mathrm{CO}_{2}$ and $95 \%$ humidity. After culturing for 7 days in the $75-\mathrm{cm}^{2}$ flask, the adherent cells (passage 0 ) were washed with phosphate-buff-

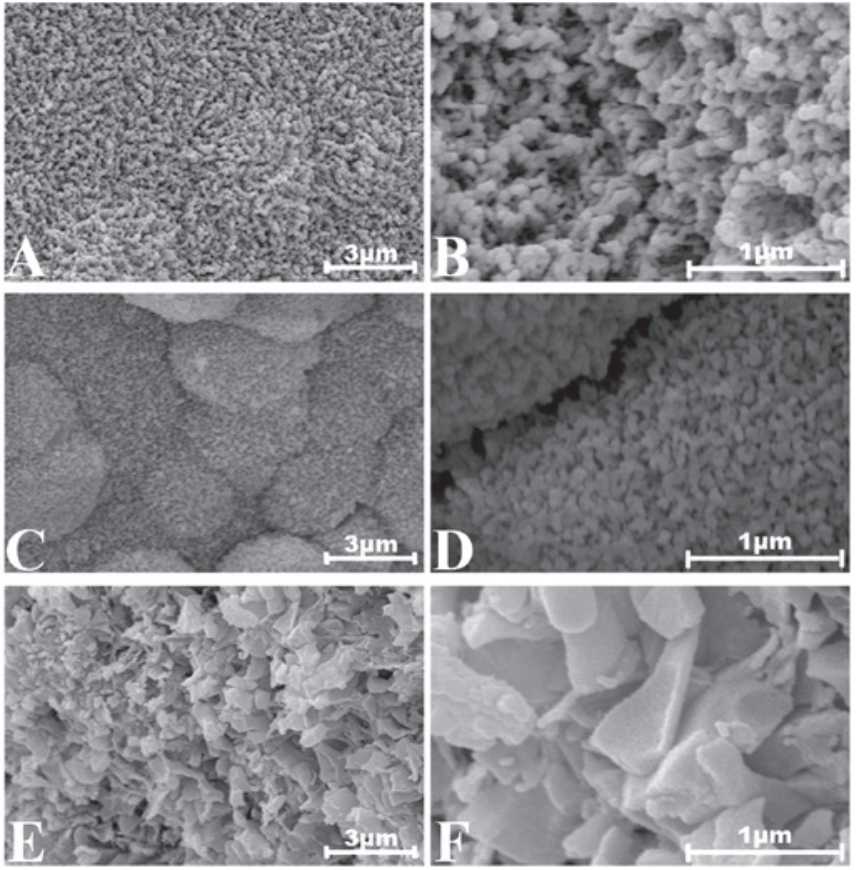

Figure 1. Scanning electron microscope (SEM) micrographs of the surfaces of the borate glass particles used in the cell culture experiments after soaking for 10 days at $37^{\circ} \mathrm{C}$ in the $0.025 \mathrm{M} \mathrm{K}_{2} \mathrm{HPO}_{4}$ solutions: (A) 3Sr group, $\mathrm{x} 10,000$; (B) 3Sr group, $x 40,000$ times; (C) 6Sr group, $x 10,000$; (D) $6 \mathrm{Sr}$ group, x40,000; (E) 12Sr group, x10,000; (F) 12Sr group, x40,000.

ered saline (PBS), and then fresh medium was added every 3-4 days. Following the initiation of cultures for 2 weeks, the cells were washed with PBS, and then lifted by incubation in $0.5 \mathrm{ml}$ of $0.25 \%$ trypsin and $1 \mathrm{mM}$ ethylenediaminetetraacetic acid for $2 \mathrm{~min}$ at room temperature. Trypsin was neutralized by adding $2 \mathrm{ml}$ complete medium. Each flask of cells was passaged into $75-\mathrm{cm}^{2}$ culture flasks every 3-4 days. After primary culturing for 2 weeks, the second passage MSCs were successfully harvested and used in this study (Fig. 2).

\section{Proliferation}

Cell Counting Kit-8 (CCK-8) assay. The extraction solutions were prepared by immersing $75 \mathrm{mg}$ partially converted glass particles (average diameter, 105-125 $\mu \mathrm{m}$ ) in $10 \mathrm{ml}$ culture medium in a $\mathrm{CO}_{2}$ incubator at $37^{\circ} \mathrm{C}$ for $24 \mathrm{~h}$, to ensure the specific surface areas reached $6 \mathrm{~cm}^{2} / \mathrm{ml}$ (according to DS/ EN ISO 10993-12:2009). Subsequently, MSCs (passage 2) were seeded in 96-well plates at a density of $1 \times 10^{4}$ cells/well in $100 \mu \mathrm{l} \mathrm{FBS}$ culture medium, in a $\mathrm{CO}_{2}$ incubator at $37^{\circ} \mathrm{C}$ for $24 \mathrm{~h}$. The medium in the well was then replaced with the 

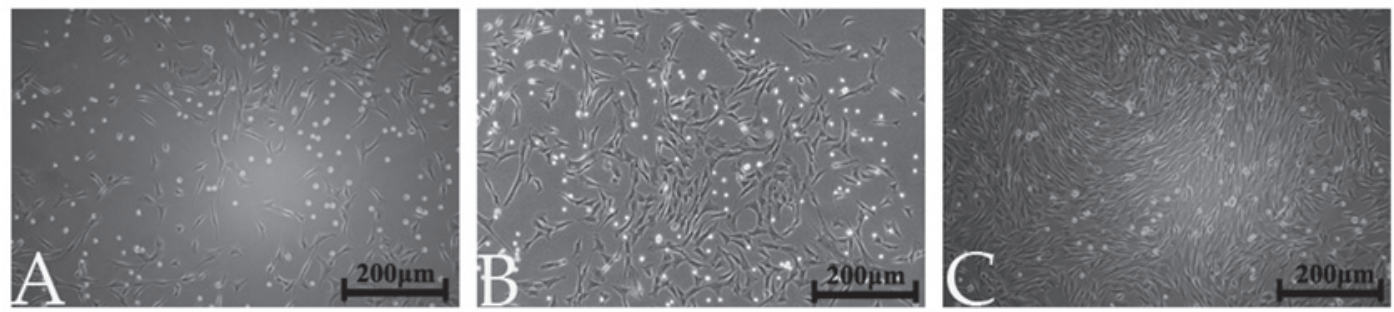

Figure 2. Photomicrographs of bone barrow-derived mesenchymal stem cells, passage 2: (A) day 1, (B) day 3 and (C) day 7.

prepared extraction solution. After 3 days, the CCK-8 (Dojindo Molecular Technologies, Inc., Rockville, MD, USA) with $10 \mu 1$ CCK- 8 was added to each well using a repeating pipettor, and then mixed on an orbital shaker for $1 \mathrm{~min}$. Following incubation for $2 \mathrm{~h}$, the absorbance of each well was measured spectrophotometrically at $450 \mathrm{~nm}$. Three parallel replicates of each sample at each time point were prepared during the cell viability assay. Statistical analyses were performed using a one-way ANOVA.

Cell morphology. The $60-\mathrm{mm}$ petri dishes with affixed partially converted glass particles $\left(105-125 \mu \mathrm{m} ; 6 \mathrm{~cm}^{2} / \mathrm{ml}\right)$ for each group (A-E, as in Table I) were placed on a small platform rocker in the $\mathrm{CO}_{2}$ incubator to gently mix in $10 \mathrm{ml}$ culture medium prior to incubation. The rocking cycle consisted of $1 \mathrm{~min}$ on and $5 \mathrm{~min}$ off. Passage 2 MSCs cells were seeded with culture medium at an initial density of 15,000 cells $/ \mathrm{cm}^{2}$ and incubated at $37^{\circ} \mathrm{C}$ in a humidified atmosphere of $5 \% \mathrm{CO}_{2}$. The phase contrast images of MSCs at the interface of each group were observed after 3 and 7 days of culturing.

Live-Dead cell staining. Similarly, MSCs cells were seeded in the medium containing each group of glass particles $\left(6 \mathrm{~cm}^{2} /\right.$ $\mathrm{ml}$ ) at a density of 15,000 cells $/ \mathrm{cm}^{2}$. After culturing for 7 days, the cells mixed with particles were rinsed with warm PBS twice and incubated for an additional $30 \mathrm{~min}$ in serum-free DMEM containing $2 \mathrm{mM}$ calcein acetoxymethyl ester (calcein AM; Biotium, Hayward, CA, USA) and $1 \mu \mathrm{g} / \mathrm{ml}$ propidium iodide (Sigma-Aldrich, USA). The fluorochrome-labeled cultures on the glass wafers were then rinsed with PBS and examined under an epifluorescent microscope fitted with appropriate exciter and emitter filters to detect live (green fluorescent) and dead (red fluorescent) cells.

DNA quantification. The global effects of the glass particles with different compositions on the proliferation of MSC cells were assessed by measuring the total cellular DNA content in cultures incubated with the glass particles in place. Triplicate samples of each glass group were added to MSC cultures in $60-\mathrm{mm}$ petri dishes and incubated for 7 days. Following incubation, the cultures were rinsed twice with PBS. The DNA on all dishes was extracted using the TIANamp Genomic DNA kit (Tiangen Biotech, Beijing, China) according to the manufacturer's instructions. The quantification of DNA concentration in each group was measured by a UV spectrophotometer (Eppendorf, Hamburg, Germany).

Differentiation. MSCs were cultured in $60-\mathrm{mm}$ petri dishes affixed with partially converted glass particles for each group (A-E, as in Table I) at an initial seeding density of 15,000 cells $/ \mathrm{cm}^{2}$. DMEM supplemented with $10 \%$ fetal bovine serum and $1 \%$ antibiotics mixture $(100 \mu \mathrm{g} / \mathrm{ml}$ of penicillin

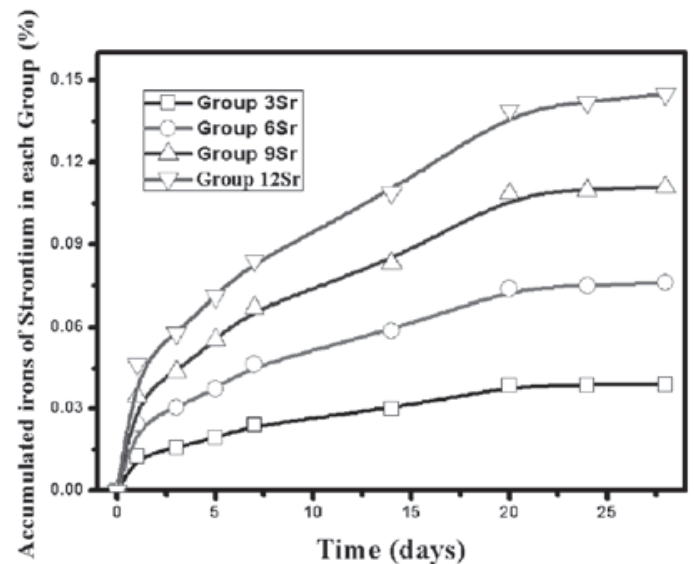

Figure 3. Accumulation of strontium leaching from the corresponding immersing solution of each group.

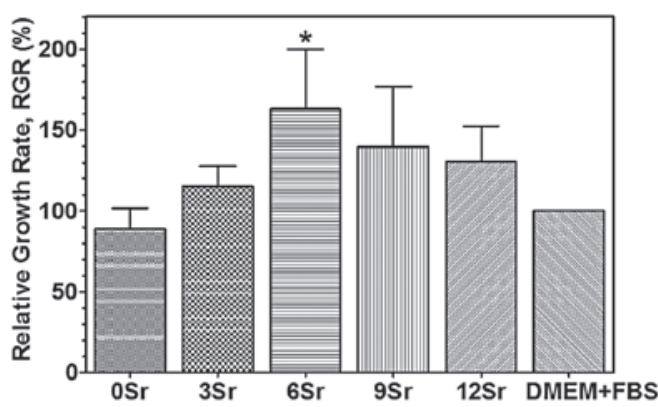

Figure 4. Relative growth rate of mesenchymal stem cells (MSCs) after culturing for 3 days with the different groups, using Dulbecco's modified Eagle's medium and fetal bovine serum (DMEM+FBS) as the control. Mean \pm standard deviation; $n=3$. "Significantly different from the DMEM+FBS group at $\mathrm{P}<0.05$.

and streptomycin) were used as the culture medium. After being grown to confluence, the culture medium of each group was replaced with osteogenic induction medium, containing $10^{-8} \mathrm{M}$ dexamethasone, $0.2 \mathrm{mM}$ ascorbic acid-phosphate and 10 mM $\beta$-glycerophosphate (Sigma-Aldrich, Co., St. Louis, MO, USA). Cells were incubated at $37^{\circ} \mathrm{C}, 5 \% \mathrm{CO}_{2}$ and $95 \%$ humidity, and the medium was replaced every 2 days.

Real-time reverse transcription-polymerase chain reaction (RT-PCR). Total RNA of each group was extracted from cells in the $60-\mathrm{mm}$ petri dishes using TRIzol reagent (Invitrogen, Carlsbad, CA, USA). Total RNA $(2 \mu \mathrm{g})$ was reverse transcribed into cDNA using the cDNA kit (Takara Biotechnology, Dalian, China) according to the manufacturer's instructions. 


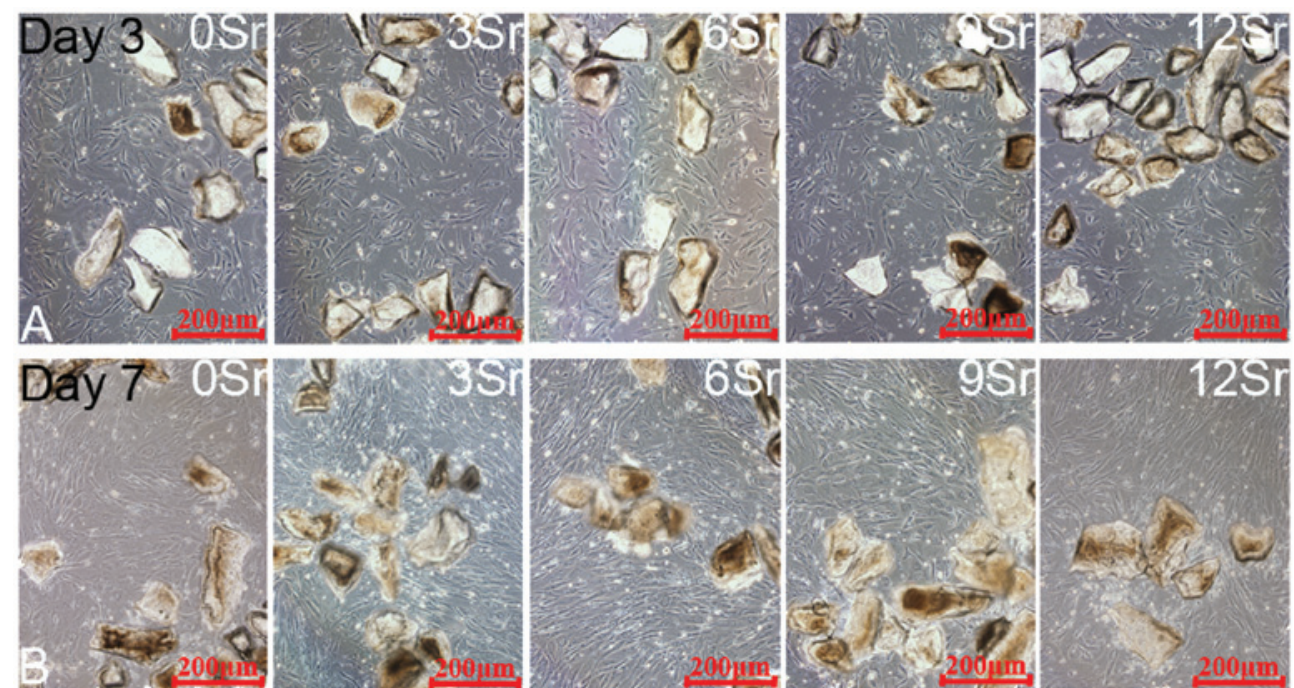

Figure 5. Photomicrographs of mesenchymal stem cell (MSC) proliferation after (A) 3 and (B) 7 days of culturing with the different groups.
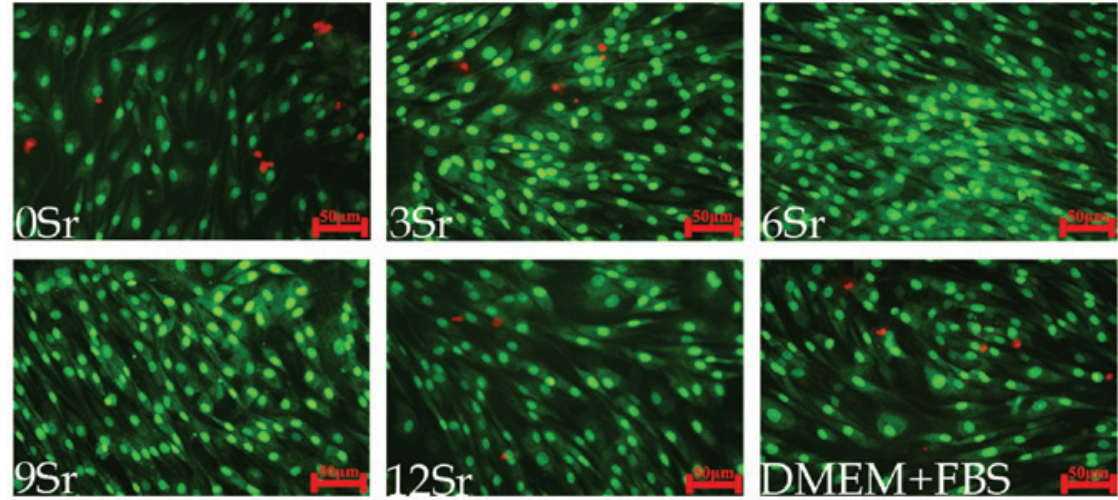

Figure 6. Live-Dead cell staining of mesenchymal stem cells (MSCs) after culturing for 7 days with each group. Living cells were detected as green fluorescence and dead cells were detected as red fluorescence.

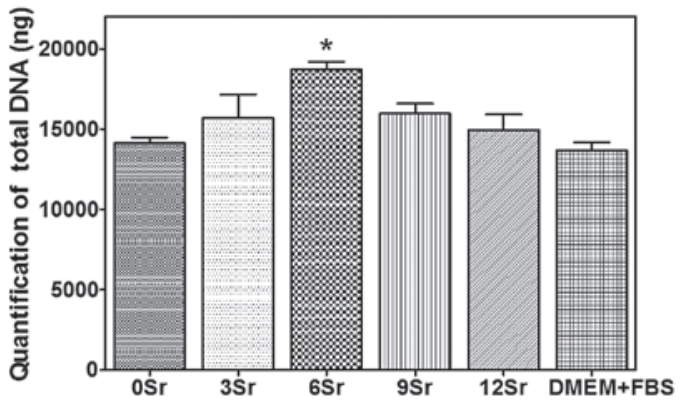

Figure 7. Quantitative measurement of DNA content in mesenchymal stem cells (MSCs) after culturing with each group for 7 days at $37^{\circ} \mathrm{C}$. Mean \pm standard deviation; $\mathrm{n}=3$. *Significantly different from Dulbecco's modified Eagle's medium and fetal bovine serum (DMEM+FBS) group at $\mathrm{P}<0.05$.

Real-time PCR was then performed to examine the expression of osteogenic genes and glyceraldehyde-3-phosphate dehydrogenase (GAPDH) mRNA levels in the MSCs. The expression levels of each gene were standardized by the internal control levels of GAPDH. Amplification was denatured at $94^{\circ} \mathrm{C}$ for $5 \mathrm{~min}$, followed by 30 cycles of denaturation at $94^{\circ} \mathrm{C}$ for $1 \mathrm{~min}$; then annealed at $54^{\circ} \mathrm{C}$ for $30 \mathrm{sec}$ and $72^{\circ} \mathrm{C}$ for $1 \mathrm{~min}$.
The temperature was then returned to $95^{\circ} \mathrm{C}$ and samples were stored at $4^{\circ} \mathrm{C}$ overnight. These procedures were performed in a PCR machine (Eppendorf).

The primer sequences used were: Forward: 5'-CCGCACGA CAACCGCACCAT-3' and reverse: 5'-CGCTCCGGCCCA CAAATCTC-3' for core binding factor $\alpha 1$ (Cbfa1); forward: 5'-CAGTAGTGACTCATCCGAAG-3' and reverse: 5'-CTCCTCTTCTTCTTCATCAC-3' for bone sialoprotein (BSP); forward: 5'-CACCGAGACACCATGAGAGC-3' and reverse: 5'-TGGTCAGCCAACTCGTCAC-3' for osteocalcin (OCN); forward: 5'-CTTTTAACTCTGGTAAAGTGG-3' and reverse: 5'-TTTTGGCTCCCCCCTGCAAAT-3' for GAPDH.

Alkaline phosphatase (ALP) activity. After 14 and 21 days of culturing, the cells were washed twice with PBS, placed in $500 \mu \mathrm{l}$ of $1 \%$ Triton X-100, and lysed using two freeze-thaw cycles $\left(-80 / 37^{\circ} \mathrm{C}\right)$. Aliquots of the lysate were placed in a 96-well plate for spectrophotometric measurement of ALP activity with p-nitrophenyl phosphate (PNPP) substrate, as previously described (17). ALP activity was normalized with respect to the total protein content obtained from the same cell lysate, and was expressed as nanomoles of PNPP formed per microgram of protein per minute. Total protein content was determined using the Micro-Bicinchoninic acid (BCA) 
A
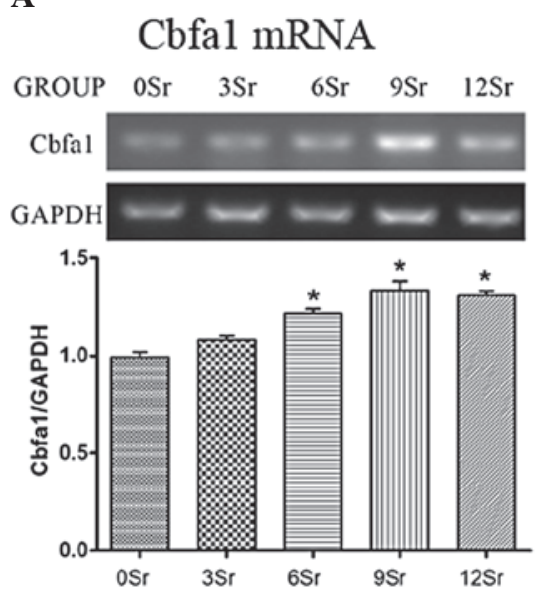

B
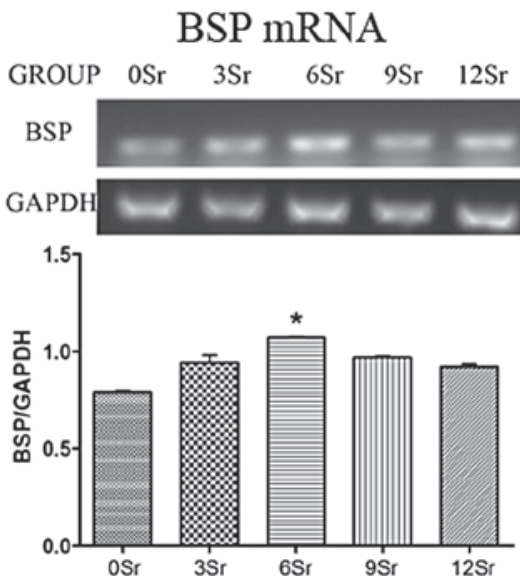

C

\section{OCN mRNA}
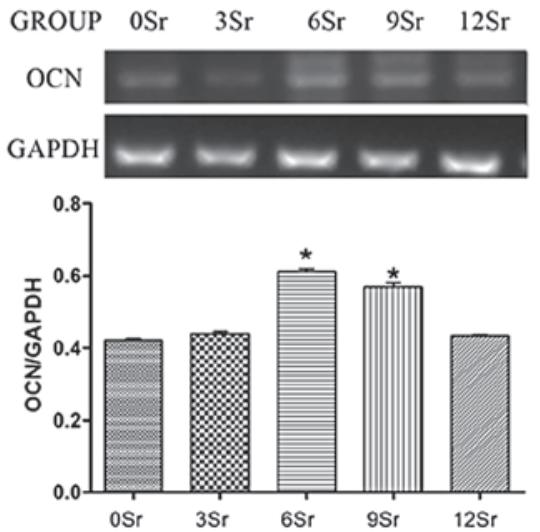

Figure 8. Expression of early, middle and late osteogenic genes in mesenchymal stem cells (MSCs). (A) Core binding factor $\alpha 1$ (Cbfa1) mRNA expression on day 7; (B) bone sialoprotein (BSP) mRNA expression on day 14; (C) osteocalcin (OCN) mRNA expression on day 21. Glyceraldehyde-3-phosphate dehydrogenase $(\mathrm{GAPDH})$ was used as the control. Mean \pm standard deviation; $\mathrm{n}=3$. *Significantly different from Dulbecco's modified Eagle's medium $(\mathrm{DMEM})$ at $\mathrm{P}<0.05$.

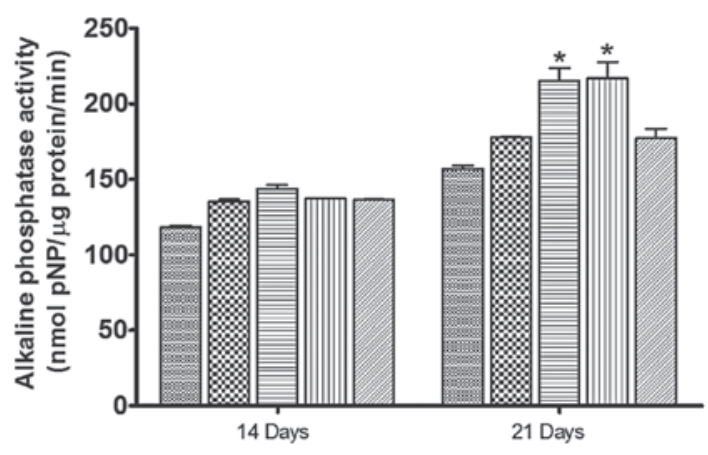

Figure 9. Alkaline phosphatase (ALP) activity in mesenchymal stem cells (MSCs) after culturing in extraction solution from each group for 14 and 21 days. Mean \pm standard deviation; $n=3$. *Significantly different from Dulbecco's modified Eagle's medium (DMEM) at $\mathrm{P}<0.05$.

Protein Assay kit (Pierce Biotechnologies, Rockford, IL, USA), according to the manufacturer's instructions.

Alizarin Red S staining. Alizarin Red S staining of MSC cultures was conducted after 21 days of culturing. The culture medium was removed from each well and cells were rinsed three times with PBS. After fixing with $4 \%$ formaldehyde solution for $30 \mathrm{~min}$ at room temperature, the cells were rinsed twice with PBS and fixed cells were stained with $1 \mathrm{ml}$ of $2 \%$ Alizarin Red S working solution (Sigma-Aldrich) and incubated for $10-20 \mathrm{~min}$ at $37^{\circ} \mathrm{C}$. Cells were then rinsed three times and visualized under an optical microscope. The number of mineralized nodules per unit was counted.

\section{Results}

\section{Sample preparation}

Characterization of degradation. During immersion in phosphate solution, the ions of strontium changed over time. As shown in Fig. 3, the concentration reached a stable level after $\geq 20$ days. From the curve, we found that in the solution, the levels of strontium released from the borate glass were relative to its composition in each group, the more strontium the particles contained, the higher the concentration in the corresponding soaking solution.

\section{Proliferation}

CCK-8 assay. Results of the CCK- 8 assay demonstrated the growth rates of the different groups relative to that of the control group with DMEM+FBS. After culturing for three days, the relative growth rates in the groups containing strontium were between 1.0 and 1.5. The $6 \mathrm{Sr}$ group demonstrated a significant difference. However, the $0 \mathrm{Sr}$ group demonstrated the lowest relative growth rate (Fig. 4).

Cell morphology. Fig. 5 shows the cell morphology (on days 3 and 7) of MSCs incubated with the strontium-containing borate glass particles of different compositions. The optical microscope revealed that all cells proliferated well along the borate glass particles. Additionally, the glass particles and the MSCs were $70 \%$ confluent, which was consistent with the results of the CCK-8 assay. On day 7, the 6Sr group showed the greatest amount of proliferation of MSCs among all the groups.

Live-Dead assay. The Live-Dead assay (Fig. 6) provided a direct observation of the proportion of living and dead cells. All the groups showed increased cell proliferation on day 7 . The $6 \mathrm{Sr}$ group exhibited the least number of dead cells and the greatest number of living cells, on staining. Other groups showed a relatively higher proportion of dead cells than the $6 \mathrm{Sr}$ group.

Total DNA concentration. Total DNA concentration, cell growth at the genetic level, indicated that the 6Sr group demonstrated the highest concentration, statistically, than any other group (Fig. 7).

\section{Differentiation}

Real-time RT-PCR. In our experiment, the expression of early, middle and late osteogenic genes in primary canine bone marrow MSCs was examined using real-time RT-PCR on days 7,14 , and 21 , respectively, to analyse MSC maturation. It was found that on day 7, compared with the 0Sr group, the Cbfal mRNA expression level was significantly higher in the 


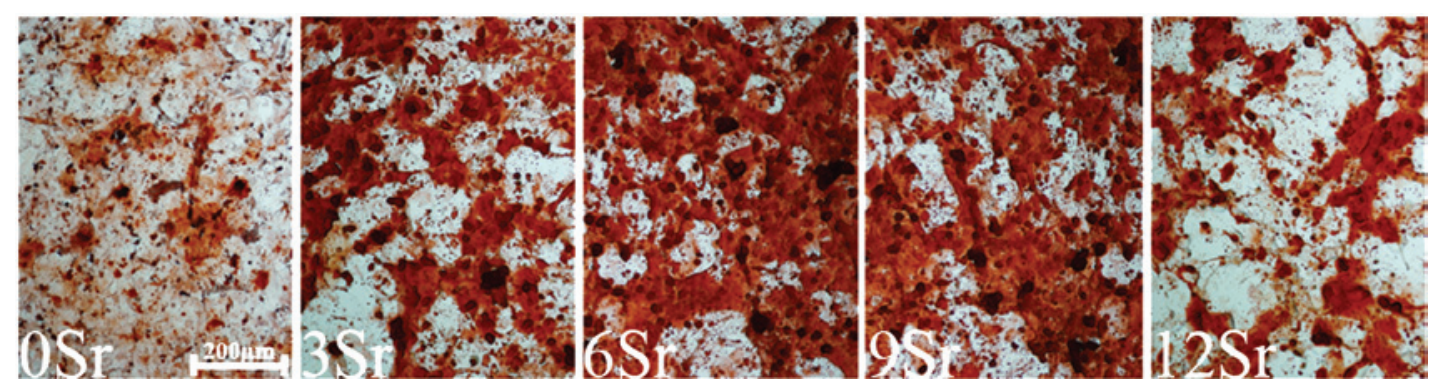

Figure 10. Optical micrographs of different groups with Alizarin Red S staining of each group after culturing with medium containing extracts of the glass particle dissolution product for 21 days.

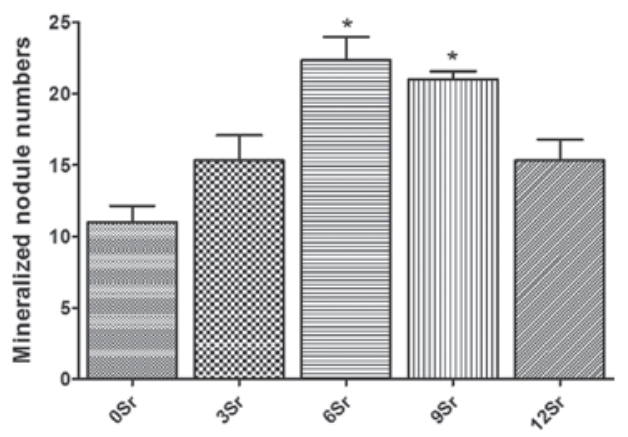

Figure 11. Mineralized nodules formation of mesenchymal stem cells (MSCs) after 21 days of culturing in extraction solution from each group. Mean \pm standard deviation; $n=3$. "Significantly different at $\mathrm{P}<0.05$.

6Sr, 9Sr and 12Sr groups $(\mathrm{P}<0.01)$. On day 14 , compared with the $0 \mathrm{Sr}$ group, the BSP mRNA level was significantly higher in the $6 \mathrm{Sr}$ group $(\mathrm{P}<0.01)$. In addition, on day 21 , similar to BSP expression, groups $6 \mathrm{Sr}$ and $9 \mathrm{Sr}$ exhibited higher $\mathrm{OCN}$ mRNA expression levels than the $0 \mathrm{Sr}$ group $(\mathrm{P}<0.01)$ (Fig. 8).

ALP activity. The results of the spectrophotometric measurement of ALP activity of MSCs in all the groups are presented in Fig. 9. On day 14, ALP levels in groups with strontium showed no significant differences compared with the 0Sr group. However, on day 21 , the $6 \mathrm{Sr}$ group demonstrated a higher ALP activity than the $0 \mathrm{Sr}$ group $(\mathrm{P}<0.01)$. Moreover, at both time points, the groups containing strontium had a higher ALP level than the 0Sr group. However, the difference was only statistically significant in the $6 \mathrm{Sr}$ group on day 21 .

Alizarin Red S staining. Alizarin Red S staining was used to examine the mineralized nodule formation by MSCs cultured with different contents of strontium-incorporated borate medium for 21 days (Fig. 10). Measured by Alizarin Red S staining, the number of mineralized nodules per unit in MSCs was also counted. The result showed that groups $6 \mathrm{Sr}$ and 9Sr had the greatest number of mineralized nodules (Fig. 11).

\section{Discussion}

Bioglass has attracted increasing attention since it was produced in $1971(3,6)$. Bioactive glasses and glass-ceramic systems are famous for their excellent biocompatibility and bioactivity in bone tissue engineering (20). After advances in industrial techniques and preparation configuration, silicate bioactive glass has gradually been replaced by borate bioactive glass, which has a wider range of bioactivity and degradation rates, with stronger bone formation in combination with hydroxyapatite for tissue regeneration. However, silicate bioactive glass has certain disadvantages: It converts slowly and incompletely to hyaluronic acid (HA) when placed in body fluid, and it is difficult to sinter 45S5 particles into a porous three-dimensional (3D) network (7). Moreover, borate-based bioactive glasses have been developed for their potential biomedical advantages (21).

Borosilicate scaffolds with the fastest degradation rate are toxic to cells, likely due to the high concentration of boron ions released into the medium (15). Increased cell proliferation has been demonstrated to occur when $<2 / 3$ of the $\mathrm{SiO}_{2}$ in $45 \mathrm{~S} 5$ glass was replaced by $\mathrm{B}_{2} \mathrm{O}_{3}$ in a borosilicate glass composition, or by partially converting the borate glass to HA prior to cell culture (17). Alternatively, dynamic culturing (17) and minor element incorporation would both achieve a biologically acceptable rate of borate release. Notably, strontium is a trace element in the human body and has been demonstrated to play a unique role in bone remodeling by stimulating bone formation and reducing bone resorption $(22,23)$. As a result, we proposed that strontium-containing borate glass particles would have a higher superficial degradation rate, and that an improved compatibility and proliferation of the MSCs may be observed.

Cytocompatibility behavior with stem cells is an important procedure to assess a novel material prior to application in tissue engineering. In the present study, all the $6 \mathrm{Sr}$ groups demonstrated a high relative growth rate after culturing for 3 days. The optical microscopy results revealed that following mixed culturing with MSC cells for 7 days, all groups showed good proliferation. However, the $6 \mathrm{Sr}$ group demonstrated the optimum growth performance, both under optical observation and the Live-Dead cell staining. It has been demonstrated that the concentration of boron released from glass incorporating strontium was 2-fold lower than that released from borate glass (16). As the radius of the strontium ion is larger than that of a magnesium or calcium ion, the strontium ion occupies more space in the glass network, and effectively inhibits the movement and release of other ions. Therefore, borate release from the glass particles can be controlled by altering the strontium oxide content in the glass composition. This explains why different compositions of strontium have different growth rates. To investigate the proliferation ability of the strontium-containing borate glass among the five different compositions of strontium, the total DNA concentra- 
tion following mixed culturing for 7 days was examined in each group. The results indicated that the 6Sr group had the highest DNA quantification, providing evidence of improved proliferation activity in this group. From a molecular biological perspective, a greater amount of proliferation activity and improved duplicate ability may be evident in the strontium-containing groups, particularly in the 6Sr group. However, the detailed underlying mechanism requires further study. Consequently, the cytotoxicity of the rapid release of boron was minimized, and incorporating strontium into borate glass may be regarded as an effective way to promote MSC proliferation.

During differentiation towards mature osteoblasts, a number of extracellular matrix genes are expressed by MSCs. For instance, Cbfa1 is a transcription factor in the early osteogenetic stage. BSP and OCN are regarded as the middle and late stage gene markers of bone formation, respectively. In the present study, the expression of these early, middle and late osteogenic genes in primary canine bone marrow MSCs was examined using real-time RT-PCR on days 7, 14 and 21 , respectively, to analyze MSC maturation. On day 7 , compared with the 0Sr group, the Cbfal mRNA level was significantly higher in groups $6 \mathrm{Sr}, 9 \mathrm{Sr}$ and $12 \mathrm{Sr}(\mathrm{P}<0.01)$. The results were consistent with a previous study that demonstrated the higher Cbfal mRNA performance of the 5-10\% strontium-substituted HA (Sr-HA) ceramics (9). Furthermore, on day 14 , compared with the 0Sr group, the BSP mRNA expression level was significantly higher in the 6Sr group. In addition, on day 21, groups $6 \mathrm{Sr}$ and $9 \mathrm{Sr}$ exhibited higher $\mathrm{OCN}$ mRNA expression levels than the $0 \mathrm{Sr}$ group $(\mathrm{P}<0.01)$. It was found that $6-9 \%$ strontium-substituted borate glass provided an improved osteogenetic ability for MSCs compared with 0-3\% strontium-substituted borate glass. It has also been demonstrated that strontium may promote the differentiation of MSCs through activation of the BSP and OCN genes in the middle and late stages of osteogenesis. However, the $12 \mathrm{Sr}$ group demonstrated poor ability in the process of bone formation, suggesting a negative feedback mechanism may affect the regulation of strontium in osteoblastic differentiation when the concentration of strontium is at a relatively higher level (9).

ALP is an important marker for osteogenic cell derivatives of MSCs. Previously, it demonstrated elevated levels when grown on 45S5 bioactive glass compared with tissue culture (24). As shown in the present study, the 6Sr group exhibited the highest ALP excretion after the 21 days of culturing. The data demonstrated that the $6 \mathrm{Sr}$ group is a reliable indication for the differentiation of MSCs into the osteoblastic lineage. Similarly, Alizarin Red S staining is an intuitionistic method to examine the mineralization capacity. In our experiments, the medium in all the groups was stained red under a microscope. It may be that the strontium-containing borate glass is able to promote MSC secretion of the osteogenetic substance. In particular, in the $6 \mathrm{Sr}$ group, the obvious mineralized nodules were observed. These results were consistent with previous in vitro studies regarding the stimulatory effect of various strontium-incorporated materials, that Sr-HA ceramics may enhance osteoblastic cell differentiation and mineralization $(25,26)$.

Strontium stimulates bone formation through its positive action on osteoblastic differentiation and function, as well as by decreasing osteoclast differentiation and function (27). The potential mechanism may involve the fact that both $\mathrm{Sr}^{2+}$ and $\mathrm{SiO}^{4-}$ ions enhance ALP activity in human bone MSCs (15). Furthermore, it has been demonstrated that strontium ranelate increases the replication of cells of the osteoblastic lineage by two distinct cell mechanisms involving CaSR, and triggering mitogenic signals, such as p38, in C3H10T1/2 cells. It has been suggested that the release of an autocrine growth factor by strontium is another potential mechanism for inducing osteoblastic cell replication (28). The detailed mechanism of strengthened bone formation by strontium requires further study.

In conclusion, borate glasses containing strontium oxide of $0,3,6,9$ and 12 mol\% demonstrate significant levels of proliferation when interacting with MSCs. The borate glass containing $6 \mathrm{~mol} \%$ strontium oxide has the best characteristic of proliferation when cultured with MSCs. The borate glass containing 6 and 9 mol\% strontium oxide facilitates improved bone formation ability compared with the remaining two compositions. Overall, it may be concluded that borate glass containing strontium provides a promising material in tissue engineering, and that $6 \mathrm{~mol} \%$ strontium oxide in borate glass could facilitate maximum proliferation and differentiation abilities.

\section{Acknowledgements}

The study was supported by the National Natural Science Foundation projects (Nos. C160802, 81000788 and 51072133). The authors would like to thank Professor Wenhai Huang (Institute of Bioengineering and Information Technology Materials, Tongji University, Shanghai, China) for his instructions and reviewing of the study.

\section{References}

1. Rahaman MN, Brown RF, Bal BS and Day DE: Bioactive glasses for nonbearing applications in total joint replacement. Semin Arthroplasty 17: 102-112, 2006.

2. Jones JR, Gentleman E and Polak J: Bioactive glass scaffolds for bone regeneration. Elements 3: 393-399, 2007.

3. Hench LL, Splinter RJ, Allen WC and Greenlee TK: Bonding mechanisms at the interface of ceramic prosthetic materials. J Biomed Mater Res 5: 117-141, 1971.

4. Hench LL and Wilson J: Surface-active biomaterials. Science 226: 630-636, 1984

5. Hamadouche M, Meunier A, Greenspan DC, et al: Long-term in vivo bioactivity and degradability of bulk sol-gel bioactive glasses. J Biomed Mater Res 54: 560-566, 2001.

6. Richard MNC: Bioactive Behavior of a Borate Glass, M.S. Thesis, University of Missouri-Rolla, 2000.

7. Fu Q, Rahaman MN, Fu H and Liu X: Silicate, borosilicate, and borate bioactive glass scaffolds with controllable degradation rate for bone tissue engineering applications. I. Preparation and in vitro degradation. J Biomed Mater Res A 95: 164-171, 2010.

8. Fu Q, Rahaman MN, Bal BS, Bonewald LF, Kuroki K and Brown RF: Silicate, borosilicate, and borate bioactive glass scaffolds with controllable degradation rate for bone tissue engineering applications. II. In vitro and in vivo biological evaluation. J Biomed Mater Res A 95: 172-179, 2010.

9. Sila-Asna M, Bunyaratvej A, Maeda S, Kitaguchi $\mathrm{H}$ and Bunyaratavej N: Osteoblast differentiation and bone formation gene expression in strontium-inducing bone marrow mesenchymal stem cell. Kobe J Med Sci 53: 25-35, 2007.

10. Yang F, Yang D, Tu J, Zheng Q, Cai L and Wang L: Strontium enhances osteogenic differentiation of mesenchymal stem cells and in vivo bone formation by activating Wnt/catenin signaling. Stem Cells 29: 981-991, 2011 
11. Peng S, Zhou G, Luk KD, et al: Strontium promotes osteogenic differentiation of mesenchymal stem cells through the Ras/MAPK signaling pathway. Cell Physiol Biochem 23: 165-174, 2009.

12. Verberckmoes SC, De Broe ME and D'Haese PC: Dose-dependent effects of strontium on osteoblast function and mineralization. Kidney Int 64: 534-543, 2003.

13. Qiu K, Zhao XJ, Wan CX, Zhao CS and Chen YW: Effect of strontium ions on the growth of ROS17/2.8 cells on porous calcium polyphosphate scaffolds. Biomaterials 27: 1277-1286, 2006.

14. Hurtel-Lemaire AS, Mentaverri R, Caudrillier A, et al: The calcium-sensing receptor is involved in strontium ranelate-induced osteoclast apoptosis. New insights into the associated signaling pathways. J Biol Chem 284: 575-584, 2009.

15. Wu C, Fan W, Gelinsky M, et al: Bioactive SrO-SiO2 glass with well-ordered mesopores: characterization, physiochemistry and biological properties. Acta Biomater 7: 1797-1806, 2011.

16. Pan HB, Zhao XL, Zhang X, et al: Strontium borate glass: potential biomaterial for bone regeneration. J R Soc Interface 7 : $1025-1031,2010$

17. Brown RF, Rahaman MN, Dwilewicz AB, et al: Effect of borate glass composition on its conversion to hydroxyapatite and on the proliferation of MC3T3-E1 cells. J Biomed Mater Res A 88: 392-400, 2009.

18. Huang W, Day DE, Kittiratanapiboon K and Rahaman MN: Kinetics and mechanisms of the conversion of silicate (45S5), borate, and borosilicate glasses to hydroxyapatite in dilute phosphate solutions. J Mater Sci Mater Med 17: 583-596, 2006.

19. Liu X, Huang W, Fu H, et al: Bioactive borosilicate glass scaffolds: in vitro degradation and bioactivity behaviors. J Mater Sci Mater Med 20: 1237-1243, 2009.
20. Rahaman MN, Day DE, Bal BS, et al: Bioactive glass in tissue engineering. Acta Biomater 7: 2355-2373, 2011.

21. Day DE WJ, Brown RF and McMenamin KD: Transformation of borate glasses into biologically useful materials. Glass Technol 44: 75-81, 2003.

22. Reginster JY: Strontium ranelate in osteoporosis. Curr Pharm Des 8: 1907-1916, 2002.

23. Buehler J, Chappuis P, Saffar JL, Tsouderos Y and Vignery A: Strontium ranelate inhibits bone resorption while maintaining bone formation in alveolar bone in monkeys (Macaca fascicularis). Bone 29: 176-179, 2001.

24. Reilly GC, Radin S, Chen AT and Ducheyne P: Differential alkaline phosphatase responses of rat and human bone marrow derived mesenchymal stem cells to 45S5 bioactive glass. Biomaterials 28: 4091-4097, 2007.

25. Ni GX, Yao ZP, Huang GT, Liu WG and Lu WW: The effect of strontium incorporation in hydroxyapatite on osteoblasts in vitro. J Mater Sci Mater Med 22: 961-967, 2011.

26. Gentleman E, Fredholm YC, Jell G, et al: The effects of strontium-substituted bioactive glasses on osteoblasts and osteoclasts in vitro. Biomaterials 31: 3949-3956, 2010.

27. Bonnelye E, Chabadel A, Saltel F and Jurdic P: Dual effect of strontium ranelate: stimulation of osteoblast differentiation and inhibition of osteoclast formation and resorption in vitro. Bone 42: 129-138, 2008.

28. Caverzasio J: Strontium ranelate promotes osteoblastic cell replication through at least two different mechanisms. Bone 42: 1131-1136, 2008. 